\title{
Insuficiencia mitral y remodelación ventricular: nuevas alternativas para su control
}

\author{
Mauricio Ortega $T^{1}$, Leonardo Gómez D² \\ 1. Médico Veterinario. Unidad de Medicina y Cirugía cardiovascular, Facultad de Medicina Veterinaria y de Zootecnia, \\ Universidad Antonio Nariño. \\ 2. Médico Veterinario MSc. Unidad de Medicina y Cirugía cardiovascular, Facultad de Medicina Veterinaria y de Zootecnia, \\ Universidad Antonio Nariño. \\ Correspondencia: mauricio.ortega@uan.edu.co \\ Recibido 31-03-08 / Aceptado 27-05-08
}

\begin{abstract}
Resumen
La remodelación ventricular es un cambio en la geometría y/o volumen de la cavidad ventricular no atribuible a cambios en la presión de distensión que produce un aumento progresivo en el tiempo del volumen ventricular. Parte del mecanismo responsable es la hipoperfusión renal, que produce una baja importante en la filtración glomerular con la consecuente activación del sistema renina-angiotensina-aldosterona, que finalmente contribuye de manera importante en la remodelación ventricular. En la actualidad, el uso de ß-bloqueadores e inhibidores de la enzima convertidora de angiotensina (IECA), constituyen una buena alternativa para evitar el proceso de remodelación en pacientes con insuficiencia cardiaca, ocasionada por un daño en el músculo cardiaco (miocarditis), daño muscular isquémico (enfermedad coronaria aguda) o por lesión valvular (endocardiosis). Como consecuencia de la degeneración valvular mixomatosa mitral (frecuente en la clínica de caninos) el paciente desarrolla insuficiencia cardiaca con remodelación ventricular izquierda que a largo plazo termina con la muerte del animal. El uso de ß-bloqueadores o de IECAS o su combinación, puede evitar en gran manera la remodelación y de esta forma evitar que se presente disfunción ventricular.

Palabras clave: angiotensina, edema pulmonar, hipertrofia ventricular, insuficiencia renal, isquemia.
\end{abstract}

\section{Abstract}

Mitral insufficiency and ventricular remodeling: new alternatives for their control

The ventricular remodeling is a change in geometry and/or volume of the ventricular cavity non attributable to changes in the distension pressure, which produces a progressive increase in the time of the ventricular volume. Part of the mechanism responsible for it is the renal hypo perfusion, which produces an important loss in the glomerular filtration with the consequent activation of the system renina-angiotensin-aldosterone, which finally has an important contribution to the ventricular remodeling. At the present time, the use of $B$-blockers and inhibitors of the angiotensin converting enzyme of (IECA) constitutes a good alternative to avoid the process of remodeling in patients with cardiac insufficiency, caused by a damage in the cardiac muscle (miocarditis), ischemic muscular damage (acute coronary disease) or by valvular injury (endocardiosis). As a result of the degeneration to valvular 
myxomatous mitral (frequent in canine clinics), the patient develops cardiac insufficiency with left ventricular remodeling that in the long term ends up with the death of the animal. The use of $\beta$-blockers or IECAS or their combination can avoid greatly their remodeling and thus avoid ventricular dysfunction from appearing.

Key Words:, hypertrophy, ischemia, pulmonary edema, renal insufficiency, ventricular angiotensin.

\section{Remodelación ventricular}

Los animales con insuficiencia mitral por lesión valvular pura, desarrollan dilatación del atrio izquierdo y del ventrículo izquierdo con la relación grosor/ radio disminuida, sin disfunción ventricular y tensión parietal aumentada. Esta sobrecarga de volúmenes tiene diferentes consecuencias anatómicas y funcionales sobre el ventrículo izquierdo (VI). Como existe regurgitación sistólica hacia el atrio, la tensión parietal inicialmente es baja y no es suficiente para generar hipertrofia ventricular izquierda (HVI), esto favorece el estiramiento de la fibra cardiaca, lo que produce dańo estructural y con el tiempo insuficiencia contráctil, por lo que aumenta el volumen sistólico, la tensión parietal y la HVI se manifiesta tardíamente $(1,2)$.

La sobrecarga volumétrica de la regurgitación mitral potencia de manera irreversible la remodelación tanto a nivel del corazón completo, como a nivel celular y molecular (3). Esta remodelación involucra varias estructuras anatómicas que incluyen las valvas, el anillo valvular, las cuerdas tendinosas, los músculos papilares y las paredes del ventrículo izquierdo.

Se conocen tres clases de remodelamiento:

1. Concéntrico cuando hay aumentos en presión.

2. Excéntrico cuando la sobrecarga es por volumen.

3. El que se genera como consecuencia de un infarto agudo de miocardio (IAM) en el que se presentan aumentos tanto de presión como de volumen.

En el remodelamiento ventricular ocurren una serie de eventos que a su vez desencadenan la liberación de sustancias que para el clínico deben considerarse como marcadores de pronóstico. La tensión parietal ventricular está directamente relacionada con los niveles plasmáticos del péptido natriurético auricular (PNA), el daño celular con la liberación de troponina y la inflamación con niveles aumentados de proteína $\mathrm{C}$ reactiva (4).
El remodelamiento ventricular ocurre por apoptosis con aumento de troponina I y $\mathrm{T}$ en ausencia de eventos isquémicos; los niveles de PNA son consecuencia de la hiperactividad neuroendocrina, que produce un aumento en la presión de la pared ventricular y forma parte activa del remodelamiento ventricular. Los niveles plasmáticos del PNA están directamente relacionados con la severidad clínica y su valoración es determinante en el seguimiento de la respuesta a la terapéutica establecida (4).

De Simone y colaboradores, reporta un trabajo realizado en la Universidad de Heidelberg sobre un estudio clínico de la geometría ventricular, dimensiones del anillo de la válvula mitral, deformación valvular y parámetros de regurgitación en pacientes con regurgitación mitral isquémica y candidatos a una anuloplastia con anillo asimétrico. Un primer grupo conformado por pacientes con enfermedad coronaria sin regurgitación mitral, un segundo grupo con enfermedad coronaria y regurgitación mitral, y finalmente un tercer grupo con regurgitación mitral por lesión valvular pura.

Los resultados muestran claramente que el grupo dos (enfermedad coronaria y regurgitación mitral) tiene los cambios más importantes tanto en las dimensiones del anillo como en la geometría del ventrículo izquierdo, un jet regurgitante menor que el del grupo tres (lesión valvular pura). Los grupos uno y tres muestran cambios en las dimensiones del anillo y de geometría ventricular menos significativos con relación al grupo dos. El paciente que sufre una mayor remodelación ventricular es aquel que padece infarto agudo de miocardio y por remodelación ventricular importante, desarrolla regurgitación mitral, que además lo lleva a disfunción ventricular y una sobrevida menor que pacientes que teniendo enfermedad coronaria isquémica, no desarrollan regurgitación (grupo uno). Esto también sugiere que la isquemia no es determinante en la remodelación y que esta puede ocurrir aún en pacientes no isquémicos (grupo tres) (5). 


\section{Sistema Renina-Angiotensina-Aldosterona (SRAA)}

El SRAA es un elemento sumamente importante en la regulación hemodinámica, así como en la de líquidos y electrolitos (6). La angiotensina II actúa sobre diferentes tejidos y células produciendo acciones diversas como la síntesis y secreción de aldosterona, liberación de catecolaminas, constricción de arteriolas eferentes renales, intercambio $\mathrm{Na}+/ \mathrm{H}+$ en el túbulo proximal, disminución del flujo coronario, disminución leve del gasto cardiaco, vasodilatación y neogénesis vascular (7). En la insuficiencia cardiaca congestiva, los niveles de renina circulantes provenientes del riñón son elevados (8). Esto se debe a un aumento de la actividad simpática aferente que llega al riñón, a una caída de la concentración de sodio en el túbulo distal y también a la disminución de la presión de perfusión arteriolar (6).

La bradiquinina es potenciada por los IECA y produce vasodilatación arteriolar, constricción arterial, constricción de venas y vénulas, liberación de oxido nítrico endotelial y depolarización causante de dolor. En la insuficiencia cardiaca congestiva, los IECA producen disminución de los niveles de angiotensina II circulantes y al potenciar los efectos de la bradiquinina producen vasodilatación periférica que reduce la poscarga y mejora el vaciamiento ventricular (7). Los IECA abaten los niveles de angiotensina II que se encuentran aumentados impidiendo su efecto (9), y a largo plazo impide la remodelación y con ello reduce el riesgo de mortalidad por insuficiencia cardiaca, arritmias e isquemia miocárdica $(10,11)$.

\section{B-bloqueadores}

La piedra angular en tratamiento de la insuficiencia cardiaca congestiva (ICC) han sido los IECA, pero otros trabajos muestran que el uso de ß-bloqueantes y antialdosterónicos también son efectivos y que reducen la morbi-mortalidad en los pacientes con ICC. Los resultados del estudio CARMEN demostraron que el uso concomitante de un $\beta$-bloqueador como el carvedilol y un IECA como el enalapril, producen una mejoría significativa en el paciente evitando la remodelación ventricular y manteniendo la función ventricular (12). De igual manera se demostró que la utilización de carvedilol sólo o en combinación con el enalapril, puede revertir el proceso de remodelamiento ventricular (12). Los ß-bloqueantes han demostrado tener un mejor efecto anti-remodelamiento con un incremento importante en la fracción de eyección (FE) en comparación con los IECA que han mostrado tener efecto sobre el remodelamiento pero un aumento mínimo de la FE (13).

La degeneración mixomatosa de la válvula mitral es una patología frecuente en ciertas razas de perros en Colombia. En muchas ocasiones el diagnóstico de la insuficiencia valvular se hace tardíamente y el animal presenta ya signos de congestión como tos, intolerancia al ejercicio, edema pulmonar etc. La importancia de un diagnóstico temprano radica en que el paciente mitral, dispara los mecanismos compensatorios con activación del SRAA por hipoperfusión renal, que como hemos visto es determinante en el proceso de remodelación y posterior disfunción ventricular izquierda. De igual manera el uso de marcadores como la aldosterona, el PNA, los niveles de troponina I y $\mathrm{T}$, son determinantes en el manejo del paciente, ya que están directamente relacionados con la severidad clínica y son de gran valor en el seguimiento de la terapia.

El remodelamiento ventricular produce cambios geométricos y/o de volumen en diferentes estructuras del ventrículo como las valvas, los músculos papilares, la pared ventricular, que alteran la hemodinamia y que traen consecuencias en la función ventricular. Este remodelamiento debe evitarse en cuanto sea posible, lo que le daría al paciente un mejor pronóstico y calidad de vida. El manejo temprano del paciente con IECA (enalapril) o con un ß-bloqueante (carvedilol, metoprolol) o su combinación, es una buena alternativa terapéutica para evitar la remodelación ventricular y evitar de esta forma el que se presente disfunción ventricular y muerte del paciente. $\mathbf{N} \mathbf{V}$

\section{Referencias}

1. Pfeffer MA, Braunwald E. Ventricular remodeling after myocardial infarction. Experimental observations and clinical implications. Circulation. 1990;81:1161-1172.

2. Guadalajara JF, Galván O, Noguera J, Alexanderson E, Cervantes JL, Huerta D. El mecanismo de remodelación en las sobrecargas de volumen del ventrículo izquierdo. Arch Inst Cardiol Méx.1995;65:217-228. 
3. Beeri R, Yosefy C, Guerrero JL, Nesta F, Abedat S, Chaput M, del Monte F, et al. Mitral regurgitation augments post-myocardial infarction remodeling failure of hypertrophic compensation. J Am Coll Cardiol. 2008;51:476-486.

4. Passino C, Poletti R, Fontana M, Vergaro G, Prontera C, Gabutti A, Giannoni A, Emdin M, Clerico A. Clinical relevance of noncardiac determinants of natriuretic peptide levels. Clin Chem Lab Med. 2008;46:1515-1523.

5. De Simone R, Wolf I, Mottl-Link S, Hoda R, Mikhail B, Sack FU, Meinzer HP, Hagl S. A clinical study of annular geometry and dynamics in patients with ischemic mitral regurgitation: new insights into asymmetrical ring annuloplasty. Eur J Cardiothorac Surg. 2006;29:355-361.

6. Garrison JC. Histamine, Bradykinin, 5 hydroxytryptamine and their antagonist. En Goodman \& Gilman`s. The pharmacological basis of therapeutics. Ed. Mc Graw Hill. 1991 pg. 749-63.

7. García D; Torruncha A. Los inhibidores de la enzima convertidora de angiotensina. Rev Cubana Cardiol Cir Cardiovasc. 1999;13:158-166.
8. Merril AJ, Morrison JL, Brannon ES. Concentration of renin in renal venous blood in patients with cronic heart failure. Am J Med. 1946;1:468-472.

9. Brown JJ, Davies DL, Johnson VW, Lever AF, Robertson JI. Renin relationships in congestive cardiac failure, treated and untreated. Am Heart J. 1970;80:329-342.

10. Agabiti-Rosei E, Muiesan ML. Influence of ACE inhibition on left ventricular hypertrophy. Br J Clin Pract. 1996;84 Suppl:17-22.

11. Kannel WB, D’Agostino RB, Silbershatz H, Belanger AJ, Wilson PW, Levy D. Profile for estimating risk of heart failure. Arch Intern Med. 1999;159:1197-1204.

12. Shimamoto K, Kawana M. The Carvedilol and ACE-Inhibitor Remodelling Mild Heart Failure Evaluation Trial Nippon Rinsho. 2007;65 Suppl:531-536.

13. Perna E.R. Tratamiento de la disfunción ventricular sistólica asintomático. Rev Med Nord. 2003;4:1-4. 